\title{
¿ES INEXISTENTE EL LEÍSMO EN CUBA?
}

\author{
Astrid Huygens \\ Universidad de Gante
}

RESUMEN: Es muy generalizada entre lingüistas del mundo hispano la opinión según la cual el leísmo - fenómeno que consiste en el uso de una forma pronominal no correspondiente a su función sintáctica - se limita al español peninsular y es ajeno al español hablado en Cuba (y en Hispanoamérica en general). Una confrontación con datos de corpus, sin embargo, nos revela que construcciones con sujeto inespecífico (p.e. las construcciones con "se" impersonal) y construcciones con sujeto inanimado (p.e. los verbos psicológicos con lectura resultativa) sí provocan la aparición de la forma dativa. Estas observaciones no encajan en las tradicionales hipótesis sintácticas. Solamente encuentran su orden si interpretamos los pronombres como un instrumento que el locutor utiliza para resaltar ciertos aspectos semánticos y pragmáticos de la frase. El sujeto inanimado y el sujeto inespecífico tienen en común la presencia del rasgo [- activo] del sujeto. La ausencia de leísmo con p.e. los verbos perceptivos nos ayuda a comprender que la actividad es un criterio de orden categórico en Cuba: un sujeto no activo va acompañado del pronombre "le", un sujeto poco o muy activo del pronombre "lo". En Andalucía, en cambio, funciona de manera gradual, por lo que podemos establecer p.e. una jerarquía de los verbos de control según su relativa accesibilidad al pronombre dativo. La baja del leísmo con los verbos de percepción con respecto a los verbos de control en esta zona nos revela que aquí el criterio operante es una combinación de los rasgos [+ activo], [+ volitivo] y [+ controlador]. Los madrileños no son insensibles a estos rasgos, pero hay otro criterio operante que tiene mucho más peso, a saber el de la topicalidad. Esto nos explica por qué en situaciones prototípicas (con relación dinámica y cohesión total entre ambos argumentos) la forma dativa es mayoritaria.

RESUMÉ: Parmi les hispanistes l'opinion selon laquelle le 'leísmo' - phénomène consistant dans l'utilisation d' une forme pronominale qui ne correspond pas à sa fonction syntaxique - se limite à l'espagnol péninsulaire et n'affecte guère l'espagnol parlé à Cuba (et en Amérique latine en général) est très répandue. Une confrontation avec les données de notre corpus montre, néanmoins, que les constructions dont le sujet n'est pas spécifié (p.e. les constructions avec " se "impersonnel) ou dont le sujet est inanimé (p.e. les verbes psychologiques à lecture résultative) provoquent bel et bien l'apparition de la forme dative. Ces observations infirment les traditionnelles hypothèses syntaxiques et n'acquièrent de sens que si l'on interprète les pronoms comme des instruments utilisés par le locuteur pour mettre en valeur certains aspects sémantiques et pragmatiques de la phrase. Ce que le sujet inanimé et le sujet non spécifié ont en commun,c'est la présence du trait [- actif] du sujet. L'absence de 'leísmo' avec e.a. les verbes de 


\section{ASTRID HUYGENS}

perception nous aide à comprendre que l'activité est un critère d'ordre catégorique à Cuba: un sujet non actif s'accompagne du pronom "le ", un sujet peu ou très actif du pronom "lo ". En Andalousie, par contre, il s'agit d'un critère graduel. Cela permet p.e. d'établir une hiérarchie des verbes de contrôle selon leur accessibilité au pronom datif. La diminution de 'leísmo' avec les verbes de perception par rapport aux verbes de contrôle nous révèle que le principe régulateur est ici une combinaison des traits [+ actif], [+ volitif] et [+ contrôleur]. Les Madrilènes ne sont pas insensibles à ces traits, mais il y a un autre trait qui a beaucoup plus de poids, à savoir celui de la topicalité. Cela explique pourquoi, dans des situations prototypiques (relation dynamique et cohésion totale entre les deux arguments), la forme dative est majoritaire.

PALABRAS CLAVE: Leísmo; gramática cognitiva; cubano; andaluz; construcciones con 'se' impersonal; verbos psicológicos.

MOTS-CLÉS: Leísmo; grammaire cognitive; cubain; andalou; constructions avec 'se' impersonnel; verbes psychologiques.

\section{Introducción}

El propósito de este artículo es doble. Es muy generalizada entre lingüistas del mundo hispano la opinión según la cual el leísmo, laísmo y loísmo - fenómenos que consisten en el uso de una forma pronominal no correspondiente a su función sintáctica - se limitan al español peninsular y son ajenos al español hablado en Cuba (y en Hispanoamérica en general). Por ser tan difundida, esta afirmación apenas ${ }^{1}$ ha sido examinada y nececista, pues, una confrontación con datos de corpus. En segundo lugar, a pesar de la enorme cantidad ya existente de estudios concernientes al leísmo, laísmo y loísmo la gramática castellana todavía no ha conseguido dilucidar de modo definitivo los criterios decisivos en la selección del pronombre 2 . Este estancamiento se debe, a nuestro entender, a que los estudios se han ocupado, hasta muy avanzados los años setenta, casi exclusivamente de los aspectos sintácticos ${ }^{3}$. El punto de partida de nuestro raciocinio no será, en consecuencia, la correspondencia entre forma y función sintáctica - o sea, no vamos a conectar por ejemplo la forma dativa a la función de objeto indirecto -, sino la "iconicidad" entre forma y contenido es decir que vamos a atribuir a cada forma unos valores semánticos y/o pragmáticos homogéneos ${ }^{4}$.

1. Hay excepciones. Véase por ejemplo Á. Di Tullio (1997)

2. Véase cómo I. Fernández Ordóñez (1993: 63) resume las investigaciones acerca de este tema.

3. Cf. É. García y R. Otheguy (1977: 65): previous studies of the use of these forms in the different dialects--and indeed the very notion of leísmo--have rested (...) on a syntactically based analysis which fails in all dialects and is therefore a poor basis for comparisons between them.

4. Nos referimos a la noción de isomorfismo, aspecto del principio de iconicidad tal como viene definido en el marco de la gramática cognitiva (cf. p.ej. M. Achard 1998: 4-5). 


\section{¿ES INEXISTENTE EL LEÍSMO EN CUBA?}

Una gran parte de este artículo consistirá por consiguiente en desechar todas las hipótesis inadecuadas acerca del leísmo, laísmo y loísmo que se han formulado, e inclinarnos, mediante la descripción de los usos recogidos en nuestro corpus, hacia una u otra opción teórica ya existente, o, eventualmente, sugerir incluso nuevos campos de investigación. El segundo propósito consiste en comprobar en qué medida el leísmo es existente en el sistema pronominal cubano y según qué principio subyacente actúa. En un artículo anterior ${ }^{5}$ estudiamos con más detenimiento las diferencias entre el sistema pronominal de Madrid, tradicionalmente considerado como centro de irradación del leísmo, y el sistema andaluz. Mediante este estudio queremos comprobar pues en qué medida el sistema andaluz desempeña el papel que tradicionalmente se le asigna de puente entre el español peninsular y el español de América.

Por consiguiente, la primera parte de este artículo pasa revista a las teorías tradicionales más importantes, como las de la R. A. E., de R. Cuervo, de R. Lapesa y otros gramáticos. Las hipótesis tradicionales se oponen a las nuevas - que estudiaremos más de cerca en la segunda parte, al analizar nuestro corpus - en que éstas prestan mucha atención a los aspectos semánticos y pragmáticos de las formas pronominales, mientras que aquéllas se centran más bien en motivos de orden etimológico, morfológico o sintáctico.

\section{Leísmo, laísmo y loísmo en la gramática tradicional}

La explicación tradicional del leísmo, laísmo y loísmo se basa fundamentalmente en dos grandes hipótesis.

La primera hipótesis consiste en lo que llamamos la hipótesis de la distinción genérica: los usos "no etimológicos" corresponderían a una tendencia a marcar bien y lisamente el género de los pronombres en lugar de su función sintáctica en la frase, como fue el caso en el latín. Mediante esta hipótesis se aclararía bien el leísmo bien el laísmo y el loísmo, según se considere respectivamente como causa principal de esta teoría genérica lo siguiente:

(a) la homonimia en el acusativo entre el "lo" masculino y el "lo" neutro ${ }^{6}$,junto con la influencia paradigmática de las formas del sujeto, de los demostrativos y del artículo,

5. cf. A. Huygens (2002)). "El leísmo: una comparativa entre Sevilla y Madrid" in: Romanistik in Geschichte und Gegenwart 8.2., 2002, p. 199 - 213

6. Cf. e.o. R. Lapesa (1968:541): Este factor es la tendencia a restablecer la distinción entre el masculino singular y el neutro, igualados por la evolución fonética de ILLUM y de ILLUD en la forma ambivalente lo.

7. Cf. e.o. J. Alcina Franch \& J. Blecua (1975:607): Masculino Femenino Neutro

el(le) ell-a ell-o

est-e est-a est-o

es-e es-a es-o

aquel-/ aquell-a aquello

l-e l-a l-o 
(b) o la confusión en el dativo entre el "le" masculino y el "le" femenino ${ }^{8}$, junto con la influencia paradigmática de las formas de los pronombres personales "me" y "te" .

La segunda hipótesis puede denominarse la oposición entre referentes animados e inanimados: aquéllos se pronominalizarían mediante el pronombre dativo, mientras que éstos llevarían la forma acusativa ${ }^{10}$. Esta distinción formaría el principio subyacente de dos causas principales del leísmo, a saber:

(a) el contagio del dativo latino a otros verbos ${ }^{11}$, ya que éstos se situarían todos en el campo de las relaciones humanas, $\mathrm{y}$

(b) el paralelismo con el acusativo preposicional ${ }^{12}$, puesto que la preposición "a" se produce únicamente ante objetos directos animados.

A estos dos grandes principios habrían coadyuvado otras sustituciones de acusativos latinos por dativos, tales como las que se produjeron en las construcciones con doble acusativo latino, con objeto directo más complemento predicativo referido a él, con verbos que pueden omitir su objeto directo, y con verbos seguidos de una cláusula infinitiva. El elemento común en estas construcciones sería que la presencia de dos elementos rigiendo el acusativo provoca la reinterpretación funcional de uno de ellos, más precisamente del acusativo con referente personal, como complemento de objeto indirecto, ya que se parte de la idea de que el verbo no puede llevar dos complementos de objeto directo a la vez. Estas construcciones habrían sido favorecedoras al leísmo - y luego por introducción de la distinción genérica al laísmo y loísmo - porque a partir de ellas estos fenómenos se habrían extendido a otros verbos, o sea que la existencia de estas construcciones rigiendo "le" donde se esperaba "lo" o "la" habría confundido a los locutores, lo cual se traduce en un uso vacilante de los pronombres con otros verbos.

8. Cf. e.o. F. Marcos Marín (1978:16): Un uso anómalo se caracterizará (...) por diferenciar el género tanto en el dativo como en el acusativo, cuando la diferencia etimológica sólo permite que se diferencie en el segundo.

9. Cf. e.o. H. Berschin (19952: 195): Als Ursache des Leismus kommt wohl in erster Linie eine Analogiewirkung der 1. und 2. Pers. in Frage: me: "mir, mich", te: "dir, dich" => le(s): "ihm, ihn, (ihnen, sie)"

10. Cf. e.o. F. Marcos Marín (1978: 17): Le se utiliza como sustituto de persona, tanto en función de objeto indirecto (dativo) como de objeto directo (acusativo, antietimológico como tal). Este uso de le como objeto directo (leísmo, en la terminología tradicional y general) opondría el rasgo (+ persona) a (-persona) en vez de la función.

11. Cf. e.o. R. Lapesa (1968: 527): El auténtico origen del leísmo fue la perduración con un grupo considerable de los verbos que en latín lo regían, y el contagio de tal construcción a otros verbos, hasta invadir toda el área del objeto directo personal.

12. Cf. e.o. A. Uruburu (1993: 51): El sintagma preposicional con a es el sustituto romance natural del Dativo latino, por lo que, al pasar a utilizarse para la función C. Directo, se produce una equiparación funcional de los dos casos y de las dos funciones, lo que puede arrastrar el uso de le, les para C. D. Como a era y es mucho más frecuente ante o para C. Directo de persona, de ahí que el primer leísmo fuera de contenido personal (...) 
Sin embargo, todas estas explicaciones presentan una limitación muy importante que I. Fernández Ordóñez (1993: 68) formula de la siguiente manera:

el leísmo en sus diferentes tipos, el laísmo y el loísmo se juzgan como fenómenos de variación porcentual sobre el total de ejemplos observados, sin que pueda predecirse qué produce su aparición en cada caso salvo "las tendencias" mencionadas.

La incapacidad predictiva se observará ante todo, como veremos, a la hora de explicar el porcentaje vacilante del leísmo según las diferentes construcciones en que se encuentra el pronombre, y la mayor o menor frecuencia del leísmo según las diferentes zonas geográficas.

\section{Crítica de la gramática tradicional}

\subsection{Construcciones con "se" impersonal}

La gramática tradicional considera las construcciones con "se" impersonal como una de aquellas construcciones contagiadas por la extensión del leísmo. F. García González (1996: 390-391) aduce el paralelismo con el acusativo preposicional como causa principal de la contaminación, y lo relaciona a su vez con con la homonimia fastidiosa con las estructuras reflexivas. Así, una frase como "se mataba el cristiano" podía significar "el cristiano se suicidaba" o "se mataba al cristiano". La preposición "a" que se introdujo a fin de evitar confusión, hizo que el complemento de persona se interpretara como un objeto indirecto y se pronominalizara mediante la forma dativa. El autor limita su hipótesis a los referentes masculinos y es ahí donde falla su teoría porque la frase "Se mataba la cristiana" es tan ambigüa como su equivalente masculino. Sin embargo, los distintos gramáticos tradicionales están de acuerdo en que para los referentes masculinos se suele recurrir a la forma dativa, mientras que para los referentes femeninos se emplea, en la mayoría de las veces, la forma acusativa. S. Fernández Ramírez (1987²: 54) ilustra esta tendencia mediante algunas cifras:

Los recuentos para le(s)/lo(s)//le(s)/la(s), en la oposición masculino// femenino, son aquí: 60/2//4//26 
Comprobemos si esta hipótesis vale para los tres sistemas pronominales ${ }^{13}$ que forman el objeto de nuestro estudio. Si hacemos abstracción del contenido semántico ${ }^{14}$ del verbo, obtenemos los promedios siguientes:

\begin{tabular}{|l|c|c|c|c|c|}
\hline CUADRO I & \% lo & \% le & & \% la & \% le \\
\hline Madrid & 15.94 & 84.06 & \multirow{5}{*}{} & 72.46 & 27.54 \\
\hline Andalucía & 4.35 & 95.65 & & 37.68 & 62.32 \\
\hline Cuba & 2.70 & 97.30 & & 8.11 & 91.89 \\
\hline
\end{tabular}

Desde entrada estos datos acaban con la supuesta ausencia del leísmo en el español de Cuba. Es más: el cubano es la variante que más leísmo presenta con este tipo de construcciones. Es también la única variante que "encaja" completamente en la teoría sobre el paralelismo con el acusativo preposicional a la que referimos antes, ya que es el única variante que exhibe porcentajes (casi) iguales de leísmo masculino (cf. ejemplo 1) y femenino (cf. ejemplo 2). Luego veremos si de verdad ésta es la explicación correcta:

1. AArnold se le llevó presurosamente hacia un montículo limpio de pasto. (CREA) ${ }^{15}$

2. Señora, espera allá afuera a que se le llame. (Trilogía sucia de la Habana. Pedro Juan Gutiérrez. Barcelona: Anagrama. 1998)

Segundo, inferimos de la clara dispersión de los datos en las zonas de Madrid y Andalucía que ésta no sólo hace imposible la explicación del fenómeno mediante la hipótesis de la distinción genérica o de la distinción entre animados e inanimados, sino que descarta cualquier explicación proveniente de una hipótesis categórica. La confusión aparente en el uso de estos pronombres requiere una explicación mediante criterios graduales. En otras palabras, la pertenencia a una categoría ("le" o "lo") no es una cuestión de sì o no, sino que se convierte más bien en una cuestión de grado. De este modo, nos inscribimos claramente en la teoría de la semántica del prototipo de G. Kleiber (1990 : 52 - 53):

Les membres d'une même catégorie n'ont plus, comme dans le modèle classique, " un degré d'appartenance plein et égal "

13. Hay que señalar que los datos cubanos son más escasos que aquellos madrileños y andaluces. Mientras que para estas zonas, disponemos para cada zona de 69 cuestionarios, para la zona cubana tenemos que recurrir a frases provenientes de periódicos y novelas. En este caso, disponemos para cada género de 44 ejemplos

14. Bien sabemos que el grado de leísmo varía según que entre los diferentes argumentos existe una relación no dinámica o dinámica, o si entre ellos hay una cohesión minimal, parcial o total. Cf. infra

15. CREA = Corpus de la Real Academia Española (http://corpus.rae.es/creanet.html) 
Un ejemplo de una hipótesis gradual nos la ofrece É. García (1977 : 67) con la noción de actividad. A partir del análisis de situaciones con tres participantes la autora atribuye a cada uno de los clíticos de tercera persona un valor semántico, lo cual, según ella, es constante, de modo que se extrapola también a situaciones de dos actuantes, donde a primera vista, podría parecer que no hay ninguna regla que rija la selección del pronombre. Los valores encontrados son los siguientes:

$$
\begin{aligned}
& l e=\text { LESS ACTIVE PARTICIPANT [con respecto al sujeto que es el más activo] } \\
& l o=\text { LEAST ACTIVE PARTICIPANT: NON FEMININE } \\
& l a=\text { LEAST ACTIVE PARTICIPANT, FEMININE }
\end{aligned}
$$

Según É. García, en situaciones de dos actuantes, el locutor, para representar un sintagma nominal masculino, puede elegir entre dos formas, a saber "lo" y "le". Si el sintagma nominal es menos activo que el sujeto pero el locutor lo considera sin embargo como bastante activo, o sea, como bastante cerca del sujeto desde el punto de vista de la actividad, será propenso a pronominalizarlo mediante "le". Si, al contrario, le atribuye al sintagma nominal poca actividad, preferirá la forma "lo". Adviértase que se trata aquí de un criterio susceptible de una interpretación personal: en algunos casos, un sintagma nominal puede ser considerado como "más activo" por algunos locutores, mientras que para otros será precisamente "menos activo".

Esta vacilación pronominal se refleja muy bien en las construcciones con objeto directo más complemento predicativo referido a él. Hay dos tipos de complementos posibles: complementos inherentes, y complementos externos. Con la noción de "externo" nos referimos a un mote, en oposición a un nombre, como en los ejemplos siguientes, sacados del corpus cubano:

3. El decía, porque yo sí que de eso no sé nada, que Machado era brujo y que llevaba esos calzoncillos en nombre de Santa Bárbara o Chango, como le llamaban los santeros. (CREA)

4. Nadie le había vuelto a llamar Turco desde que perdiera el nombre en una apuesta de gallos, allá en un palenque de Key West, y cambiara el oficio de domador de cascadas por el de domador de leones. (CREA)

Por razones prácticas de corpus restringimos nuestra investigación a los complementos externos. El CUADRO II exhibe los resultados de los recuentos ${ }^{16}$ :

16. Cf. nota 13. Para esta construcción disponemos de 42 ejemplos cubanos (todos provenientes del CREA y de novela Trilogía sucia de la Habana 


\begin{tabular}{|c|c|c|c|c|c|c|}
\hline \multirow{3}{*}{$\begin{array}{c}\text { CUADRO } \\
\text { II }\end{array}$} & \multicolumn{2}{|c|}{ Madrid } & \multicolumn{2}{|c|}{ Andalucía } & \multicolumn{2}{|c|}{ Cuba } \\
\hline & $\%$ lo & $\%$ le & $\%$ lo & $\%$ le & $\%$ lo & $\%$ le \\
\hline & 36,23 & 63,77 & 33,33 & 66,67 & 54,55 & 45,45 \\
\hline
\end{tabular}

La vacilación es muy pronunciada en Cuba, donde los resultados se dividen casi en un 50 por 100. Tomando en cuenta la teoría de la relativa actividad de É. García podemos explicar la aparente variación libre de la manera siguiente: con las construcciones con objeto directo más complemento predicativo cabe duda acerca del número de participantes por lo que la diferenciación entre los papeles semánticos (el más activo/menos activo/el menos activo) se vuelve borrosa, en oposición a las situaciones de tres participantes donde la distribución es extremamente nítida:

Nicknames or ad hoc designations do not force a le: lo is also possible, since after all the nickname or designation can be identified with the person named, thus reducing the situation to two participants. É. García (1975: 295)

En la construcción con "se" impersonal, el participante más activo es "se", pero al mismo tiempo parece borrado de la frase, ya que uno recurre a una frase impersonal con "se" cuando no quiere expresar, o cuando no quiere expresar precisamente, quién ha realizado la acción en cuestión. La ausencia semántica del sujeto hace crecer el objeto en importancia, y lo convierta en el participante más activo $^{17}$. De este modo adopta más fácilmente la forma dativa, siendo ésta índice de un grado de participación elevada. I. Fernández Ordóñez (1993: 71) apunta otro tanto:

Por otro lado, si el sujeto no es tan "activo" como sería de esperar, el objeto estará vinculado a la acción verbal y también podrá ser referido por le. El sujeto es débilmente activo si es inanimado (...), o es inespecífico (lo fusilaron se le fusiló).

El criterio de la actividad demuestra su validez para explicar el leísmo cubano en las dos construcciones estudiadas hasta ahora. También resulta satisfactorio para aclarar el leísmo masculino en Madrid y en Sevilla. En estas regiones, sin embargo, hay que afinarlo porque no dilucida la baja del leísmo con referentes femeninos. El argumento basado en el machismo de la sociedad ibérica invocada e.o. por É. García (1975: 330) no nos satisface:

17. Cf. observado también por N. Delbecque. \& B. Lamiroy (1996) 
It is furthermore also important to stress that the norm of leísta dialects is not arbitrary: it is true that males are inherently more active than females, and it cannot be questioned, moreover, that whithin Spanish culture (if not universally), males are socially more highly valued than females.

Veamos a través del estudio de algunas construcciones más si el argumento de la activad se mantiene en Cuba y cómo podemos ajustarlo a los datos andaluces y madrileños.

\subsection{Construcciones con verbos de influencia seguidos de un infinitivo}

La primera construcción que nos ayudará en esta tarea de perfilación de la teoría avanzada es la con el verbo de influencia seguido de un infinitivo. Los resultados para Andalucía y Madrid ya están reproducidos en A. Huygens (2002) pero completémolos ahora mediante aquellos cubanos ${ }^{18}$ :

\begin{tabular}{|c|c|c|c|c|c|c|}
\hline \multirow{2}{*}{$\begin{array}{c}\text { CUADRO } \\
\text { III }\end{array}$} & \multicolumn{2}{|c|}{ MADRID } & \multicolumn{2}{c|}{ ANDALUCÍA } & \multicolumn{2}{c|}{ CUBA } \\
\cline { 2 - 7 } & \%lo & \%le & \%lo & \%le & \%lo & \%le \\
\hline Ayudar & 26.09 & 73.91 & 14.49 & 85.51 & 72.22 & 27.78 \\
\hline Obligar & 20.29 & 79.71 & 18.84 & 81.16 & 90.00 & 10.00 \\
\hline Mandar & 24.64 & 75.36 & 23.19 & 76.81 & 100.00 & 0.00 \\
\hline Invitar & 23.19 & 76.81 & 30.43 & 69.57 & 86.11 & 13.89 \\
\hline Enseñar & $/$ & $/$ & $/$ & $/$ & 8.00 & 92.00 \\
\hline
\end{tabular}

Discutamos en primer lugar los porcentajes provenientes de Cuba. A primera vista no se deja discernir una lógica ninguna. No obstante, al contemplar los casos con forma dativa de los dos primeros verbos, constatamos que en todos ellos tenemos un sujeto inanimado:

5. Este gusto por el estudio sistemático de la literatura y el vocabulario, que en su [=del catedrático] primera juventud había resultado un agradable pasatiempo (en varias millas a la redonda era famoso por el arte de solucionar crucigramas), había devenido una forma de obsesión por el idioma que para él tenía el mismo valor que Goldwyn Mayer para Tartufo o las palomas para Blas Adán o la caja de terciopelo para Asdrúbal: el valor inapreciable de ayudarle a sobrellevar la fatalidad. (CREA)

6. - Algunos bailarines habían abandonado la farsa para comer panqueques y natillas, que los servidores de Palacio les ofrecían en bandejas, pero el sonsonete de la gaita y el chillido de los cornetines, el golpeteo de los tamboriles y los soniquetes de chirimías y pífanos, unidos a la algarabía de las comparsas y la explosión de triquitraques y petardos, les obligaba a volver al baile. (CREA)

18. Cf. nota 13. Para el verbo "ayudar" tenemos 54 ejemplos cubanos, para el verbo "obligar" 40, para el verbo "mandar" 36, para el verbo "invitar" 42, y para "enseñar" 50. 
Los sujetos de estas frases son inanimados lo que lleva al locutor a atribuir el papel semántico del participante más activo al objeto de la frase. La pronominalización se realiza entonces mediante la forma dativa. El verbo "mandar" confirma esta hipótesis ya que los ejemplos de los que disponemos llevan todos un sujeto animado lo que justifica el $0 \%$ de leísmo con este verbo. Los casos de "le" (ejemplo 7 y 8) con el verbo "invitar", al contrario, se resisten a esta hipótesis, visto que todos llevan un sujeto animado:

7. Tomás Fairfax, el hijo del general Fairfax, entró al comedor saludando a los presentes; Leven le invitó a compartir la cena pero se disculpó porque había comido temprano (CREA)

8. Al rato, el político le invitó a beber cerveza en un café cercano, y al pasar junto al supuesto mendigo, el payaso comprendió que Asdrúbal era Asdrúbal. (CREA)

9. El mago los invitó a recorrer la choza y comprobar por sí mismos lo que les decía. A la orden del coronel, dos cazadores saltaron a tierra (CREA)

Algunos han avanzado la presencia del objeto directo para explicar el dativo en estos casos, pero la frecuencia elevada con la que encontramos frases del tipo del ejemplo 9 falsea la validez de este argumento sintáctico. Lo único que se nos ocurre es que estamos ante un fenómeno de gramaticalización que tiene lugar cuando "invitar" se combina con un verbo de comer o de beber. Sin duda también cabe hablar de gramaticalización en el caso de "enseñar": tan sólo detectamos cuatro formas acusativas, mientras que en todos los demás ejemplos tenemos una forma dativa (ejemplo 10). Incluso encontramos un ejemplo en el que el referente es un animal (ejemplo 11):

10. Por un momento pensó darles por dormitorio el aula de biología humana, por aquello de que tuvieran buenos puntos de referencia a la hora de conocer los secretos de sus cuerpos, pero se acordó de la tarde que la mataron de amor a cielo abierto en un rancho triplerre de California, Dios te guarde, vaquero, y reconsideró la propuesta, convencida de que los jóvenes sabrían encontrar, entre tanta fiera insípida, la serpiente de cascabel que les iba a enseñar a pecar como es debido. (CREA)

11. Entonces cita, y la justifica, aquella anécdota de La Bruyére, de un señor que le pagaba a un maestro de órgano para que $\mathbf{l e}^{19}$ enseñase a cantar a sus canarios, mientras se preocupaba muy poco por la educación de sus hijos. (CREA)

19. Es bastante frecuente en el español de Cuba encontrar la combinación de un objeto indirecto plural y un pronombre singular. 
Queda ahora por contemplar los porcentajes de leísmo en Andalucía y en Madrid. Como ya observamos en A. Huygens (2002), constatamos que en Madrid el porcentaje de "lo" queda atascado en los cinco casos en un mismo promedio. En cambio, en Andalucía se pueden apreciar grandes diferencias entre cada una de las frases. Así podemos dar cuenta de la accesibilidad al pronombre dativo según la jerarquía siguiente:

ayudar $>$ obligar $>$ mandar $>$ invitar

Esta jerarquía confirma la validez del criterio de la relativa actividad para el leísmo en Andalucía. Actúa también en Madrid (sino los porcentajes de leísmo en esta región no serían tan parecidas a los andaluces) pero de manera más suave.

\subsection{Construcciones con verbos de percepción seguidos de un infinitivo}

He aquí los resultados respecto ${ }^{20}$ a los verbos de percepción visual ${ }^{21}$, concretamente al verbo "ver":

\begin{tabular}{|c|c|c|}
\hline CUADRO IV & \% lo & \% le \\
\hline Madrid & 30.43 & 69.57 \\
\hline Andalucía & 47.83 & 52.17 \\
\hline Cuba & 87.88 & 12.12 \\
\hline
\end{tabular}

Llama la atención el bajo porcentaje de leísmo en Cuba. La mayoría de las veces se recurre en efecto al pronombre acusativo para pronominalizar el complemento del verbo de percepción (cf. ejemplo 12 y 13):

12. Esa noche Santico no viene. Ella lo ve pasar entre los bejucos del monte. (Trilogía sucia de la Habana. Pedro Juan Gutiérrez. Barcelona: Anagrama. 1998)

13. Estaba más desencantado aún que Fernando pero se concebía a sí mismo como un hombre sin futuro y le horrorizaba la idea de morir fuera de Cuba. En eso, lo vi acercarse. (CREA)

20. Cf. nota 13, para "ver" disponemos de 66 ejemplos. En las tres regiones se trata de ejemplos sin objeto directo. En A. Huygens (2002: 206) ya observamos que el leísmo no varía según que el verbo de percepción lleva un objeto o no

21. Se pueden apreciar ligeras diferencias entre el leísmo con verbos de percepción visual y el leísmo con percepción auditiva, cf. E. Roegiest (2000: 6) 
Esto no infirma necesariamente la validez de la opción teórica que hasta aquí propusimos para Cuba. Conviene averiguar donde residen las mayores diferencias entre los verbos de percepción y las construcciones antes examinadas. En el caso de la construcción con "se" impersonal el sujeto de la frase estaba semánticamente ausente, por lo que el objeto se convirtió en participante más activo, mientras que en el caso de los verbos de control, la forma dativa se debió a la presencia de un sujeto inanimado. Ambos casos tienen en común el rasgo "débilmente activo" del sujeto. Aquí tenemos una situación distinta. Hay un sujeto (en la frase 12 es "ella", en la frase 13 es el sujeto de "se concebía") (/v/ construcción con "se" impersonal) y el sujeto es animado (/v/ construcción con verbo de control). Estos dos criterios bastan para que el cubano no dude acerca del número de participantes (/v/ construcción con complemento predicativo) y atribuya, sin darle muchas vueltas al tema, el papel de participante más activo al sujeto, y el papel de participante menos activo al objeto. El grado de actividad de este sujeto parece, pues, tener poca importancia.

Por lo concerniente a los resultados andaluces, se desprende del cuadro IV una baja de leísmo con respecto a los verbos de control. Como ya explicamos en A. Huygens (2002) esto se debe fundamentalmente a dos razones:

1. La ausencia del rasgo [+ volitivo] que sí está presente en los verbos de control. El sujeto del verbo "ver" no tiene la voluntad de ver. Es más algo que se le ocurre. Al contrario, "obligar/mandar/ayudar... alguien a hacer algo" es algo querido por el sujeto de estos verbos.

2. Cabe duda acerca del rasgo controlador del sujeto. Por un lado, comprobamos que un verbo como "ver" no controla la acción expresada por el infinitivo en la misma medida que lo hace por ejemplo "enseñar", de forma que podemos concluir que el objeto es relativamente independiente y, por ende, poco controlado por la acción del sujeto. Por otro lado, tenemos que reconocer, como apunta E. Roegiest (2000: 3-4), que:

le caractère non statique de la perception directe (...) permet d' attribuer une propriété causale non au stimulus mais au percepteur qui par là assume un certain degré de contrôle sur la perception. Les $V$ de perception directe exercent d'ailleurs, toujours selon la théorie de C. Felser (1999: 6), un contrôle spécifique sur l' objet, ce qui est corroboré par la contrainte de la simultanéité temporelle.

Como se puede observar, nos vemos obligada, para la región de Andalucía, a recurrir, al lado del rasgo de la relativa actividad, a otros criterios para explicar el descenso del leísmo con verbo de percepción con respecto a los verbos de control, a saber el carácter no volitivo y poco controlador del sujeto más el objeto poco 
afectado. Esto nos invita a considerar una sustitución de la teoría de É. García por una teoría más elaborada. La propuesta de D. Dowty (1991), luego afinada por B. Primus (1999: 36-38) nos parece atractiva. Este autor divide la noción de actividad en los siguientes componentes: volitional involvement (implicación volitiva), sentience (facultad perceptiva), causing (causativo), movement (movimiento). La noción de pasividad implica entonces: change of state (cambio de estado), incremental theme (tema incremental), caused (causado), stationary (estacionario). Un tema incremental implica que el argumento cambia de estado y que es posible distinguir las distintas etapas de este cambio.

Finalmente, en cuanto a la zona madrileña el porcentaje del leísmo con verbos de percepción (+/- $70 \%$ ) corresponde más o menos a los porcentajes medios reproducidos en los cuadros anteriores. Esta observación apunta en la dirección de una hipótesis "referencial": la selección del pronombre viene determinada por la naturaleza semántica del referente y resulta insensible a la posible influencia de elementos sintácticos o a la naturaleza del verbo o del sujeto.

Los datos de los verbos psicológicos que examinaremos en el párrafo siguiente nos revelarán si estos criterios son suficientes para explicar todos los aspectos del leísmo.

\subsection{Construcciones con verbos psicológicos}

N. Ruwet (1971) divide los verbos psicológicos en dos subcategorías, según seleccionan obligatoriamente bien un sujeto bien un objeto humano. En el primero caso (ejemplo 2214 y 15), se trata de verbos tales como "desdeñar", "amar", "adorar", "admirar", "detestar" etc. mientras que en el segundo caso tenemos verbos del tipo "disgustar", "divertir", "interesar", "aburrir" etc. La segunda categoría se divide a su vez en dos subcategorías según estamos ante una lectura agentativa (ejemplo 16 y 17) o una lectura resultativa (ejemplo 18 y 19):

14. Cuando su marido murió, ella tenía sesenta y tres. Él, noventa y cuatro. Fue un médico reconocido en La Habana. Jamás lo amó. Jamás le gustó físicamente. (Trilogía sucia de la Habana. Pedro Juan Gutiérrez. Barcelona: Anagrama. 1998)

15. Era tornero y ganaba bastante. Lo suficiente para mantener a su esposa y tres hijos y a Carmita con sus dos niños. Sólo existía una dificultad: Carmen no lo soportaba. (Trilogía sucia de la Habana. Pedro Juan Gutiérrez. Barcelona: Anagrama. 1998)

22. Los ejemplos salen todos del corpus cubano 


\section{ASTRID HUYGENS}

16. ¿Usted es amigo del Rafael ese? - No, lo conocí por casualidad. - Es un anormal. Lo sorprendimos violando el cadáver de una mujer. (Trilogía sucia de la Habana. Pedro Juan Gutiérrez. Barcelona: Anagrama. 1998)

17. El condenado sonrió a los niños y éstos rieron con él, y en seguida se marcharon a buscar otra diversión porque no lograban irritarlo. (CREA)

18. El nombre del plomero es Pancracio. A él no le importa. No es que no le importe. Es que no percibe lo ridículo de ese nombre. (...) Pero su oficio de plomero es lo que más le gusta. No sabe por qué ni le interesa saberlo. Le gusta. (Trilogía sucia de la Habana. Pedro Juan Gutiérrez. Barcelona: Anagrama. 1998)

19. El otro momento importante fue cuando se casó (=Pancracio). Ése fue un día feliz, pero a la semana siguiente comenzaron las broncas con su mujer y se separaron en una semana. Desde entonces no le interesa nada. Pero eso ya ni el sexo le atrae. (Trilogía sucia de la Habana. Pedro Juan Gutiérrez. Barcelona: Anagrama. 1998)

Á. Di Tullio (1997) pretende que en el español de Hispanoamérica la forma acusativa corresponde a la lectura agentiva, y la forma dativa a la resultativa. Si esto fuera así, los verbos psicológicos ratificarían los resultados anteriores, y especialmente los de los verbos de control con sujeto inanimado. Echemos un vistazo al cuadro $\mathrm{V}^{23}$ :

\begin{tabular}{|c|c|c|c|c|c|c|}
\hline $\begin{array}{c}\text { CUADRO } \\
\text { V }\end{array}$ & \multicolumn{2}{|c|}{ Categoría 1 } & \multicolumn{2}{c|}{$\begin{array}{c}\text { Categoría 2 } \\
\text { lectura agentiva }\end{array}$} & \multicolumn{2}{c|}{$\begin{array}{c}\text { Categoría 2 } \\
\text { lectura resultativa }\end{array}$} \\
\hline & \%lo & \%le & \%lo & $\% l e$ & $\% l o$ & $\% l e$ \\
\hline Madrid & $/$ & $/$ & 11.59 & 88.41 & 1.45 & 98.55 \\
\hline Andalucía & $/$ & $/$ & 4.35 & 95.65 & 2.90 & 97.10 \\
\hline Cuba & 84.62 & 15.38 & 92.31 & 7.69 & 9.68 & 90.32 \\
\hline
\end{tabular}

Como ya lo dejaban entrever los ejemplos (14 - 19) antes enumerados, los resultados cubanos encajan perfectamente en la teoría antes expuesta para esta región.

23. Cf. nota 13. No tenemos datos acerca de la primera categoría de verbos psicológicos para las regiones de Madrid y Andalucía. Para Cuba tenemos 78 ejemplos para categoría 1, 78 ejemplos también para la lectura agentiva, y 62 para la lectura resultativa. Otra vez hacemos abstracción del contenido semántico del verbo, cf. nota 14 
Mientras que en Cuba hay una discrepancia brutal entre el promedio con sujeto animado y el promedio con sujeto inanimado ${ }^{24}$, en Andalucía es mínima. J. Van Voorst (1993a) observa que el rasgo fundamental de los verbos psicológicos de la segunda clase es la ausencia del control por parte del sujeto sobre el objeto. N. Ruwet (1994), en cambio, enfoca ante todo la relativa actividad del sujeto: para reaccionar a una emoción hay que percibirla primero. Ambas características apuntan en la misma dirección: el sujeto es menos activo de lo normal, y el objeto es más activo de lo que debería. La distancia entre ambos participantes se achica y resulta en la aparición del dativo.

En Madrid estamos otra vez ante una diferencia considerable entre el leísmo con sujeto animado (lectura agentiva) y sujeto no animado (lectura resultativa). Esto no nos debe sorprender. Más arriba ya sugerimos que en la selección del pronombre los madrileños se dejan guiar ante todo por la naturaleza semántica del referente y son más bien insensibles a la posible influencia de elementos sintácticos o a la naturaleza del verbo o del sujeto. El referente de la frase con lectura agentiva es más prominente que la frase con lectura resultativa: un sintagma nominal humano atrae con más facilidad la atención sobre sí que un sintagma nominal inanimado. Más el estímulo aparece como prominente, menos probable será la ocurrencia de "le".

\section{Conclusión}

Con el leísmo, uno de los caballos de batalla de la gramática castellana, estamos aparentemente ante una variación libre: se pueden encontrar con facilidad frases donde el referente masculino se pronominaliza dos veces mediante un pronombre distinto, a pesar de que desempeñe dos veces la misma función sintáctica de objeto directo en la frase.

El porcentaje de casos de leísmo, laísmo y loísmo que las teorías sintácticas dejan inexplicados, y que, en primera instancia, aparecen ante nosotras de forma caótica, encuentran su orden y concierto al interpretarlos como un instrumento que el locutor utiliza para resaltar aspectos semánticos y pragmáticos concretos del significado de las palabras y expresiones, tal y como se le aparecen esos conceptos en su interpretación personal. No obstante, lo que hemos llamado "interpretación personal", es evidente que no es tan personal en todo los casos, y que, por algunos motivos, determinados conceptos de las cosas se generalizan en ciertas zonas y

24. D. Bouchard (1995) observa que la lectura resultativa no siempre implica un sujeto inanimado. También puede ser animado, pero en este caso no se refiere tanto al individuo, sino a ciertas propiedas de este individuo. Cf. Paul m'a frappé par inadvertance (lectura agentiva) /v/ Paul m'a frappé par son intelligence (lectura resultativa) 
con ellos el uso del los pronombres asociados. Resulta pues posible especificar cuáles son los factores semánticos y pragmáticos que intervienen en la selección del pronombre en Madrid y cuáles actúan en Andalucía y cuáles guían los locutores cubanos.

Los cubanos son los que menos recurren al leísmo. Sólo lo hacen cuando el sujeto de la frase "desaparece" bien porque es inespecífico (en el caso de la construcción con "se" impersonal) bien porque es inanimado (en el caso de los verbos de control con sujeto inanimado y en el caso de los verbos psicológicos del segundo tipo con lectura resultativa). En todos los demás casos, por muy poco activo que el sujeto sea (por ejemplo en el caso de los verbos psicológicos del primer tipo), el objeto se pronominaliza mediante la forma acusativa. De esto se desprende que el criterio operante está claramente relacionado con la actividad del sujeto. La actividad de los participantes condiciona también (al menos parcialmente) el leísmo andaluz. Sin embargo, hay una diferencia muy importante entre los dos sistemas pronominales: los andaluces son sensibles a los distintos niveles de actividad, los cubanos no: si el sujeto es inactivo el objeto asumirá el papel de participante más activo y se pronominalizará mediante "le", si el sujeto es activo (en el sentido de que realiza una acción, aunque si ésta no requiere ningún tipo de esfuerzo) el objeto se considera como menos activo que el sujeto y se pronominalizará mediante "lo".

Los jóvenes andaluces recurren bastante frecuentemente al leísmo. En esto son sensibles a factores de orden semántico: el grado de actividad, como lo muestran claramente los verbos de control, pero también el grado de control y el carácter volitivo o no, como nos reveló el análisis de los verbos perceptivos y los verbos psicológicos. El conjunto de estos rasgos, [+ activo], [+ controlador] y [+ volitivo] forman el proto-agente de D. Dowty. Cuanto más el proto-agente se acerca al proto-paciente (que se caracteriza por los rasgos opuestos) más la aparación del pronombre dativo se vuelve probable.

Los madrileños suelen generalizar más el uso de "le", basándose "grosso modo" en los mismos principios, pero añadiendo un aspecto más bien pragmático: la prominencia, el grado de autonomía referencial, sea del objeto sea del sujeto parece influir en la ocurrencia de "le". Es este criterio que permite comprender el bajo porcentaje de leísmo femenino: en la mente humana los entes masculinos están presentes antes que los entes femeninos.

El sistema pronominal andaluz constituye un puente entre la variante madrileña y la variante cubana en que reúne características de ambas: por una parte actúa - aunque de manera más atenuada que en Madrid - el criterio de la prominencia, y por otra actúa - aunque de manera más rafinada - el criterio de la actividad. En Andalucía la actividad es un criterio de naturaleza gradual, en Cuba es un criterio categórico. 
Como los aspectos considerados son varios y pueden presentarse juntos en un mismo contexto y como depende del locutor la ponderación de los mismos, siempre queda abierta la posibilidad de que alguien rompa la norma de la zona. Esto es, si no sabemos con exactitud cuál es el peso que cada locutor da a los distintos factores, puede aparecer un "le" donde esperábamos un "lo" o un "la".

\section{Bibliografía}

ACHARD, M. Representation of Cognitive Structures. Syntax and Semantics of French Sentential Complements. Berlin, New York: Mouton de Gruyter, 1998.

ALCINA FRANCH, J. y J. BLECUA. Gramática española, Barcelona: Ariel, 1975. BERSCHIN, H.; J. FERNÁNDEZ-SEVILLA y J. FELIXBERGER. Die Spanische Sprache. Verbreitung, Geschichte, Struktur. Ismaning: Hueber, 1995.

BOUCHARD, D. "Psych Constructions and Linking to Conceptual Structures". En: HIRSCHBÜHLER, P. y K. KOERNER (eds.). Romance languages and modern linguistic theory. Amsterdam, Philadelphia: John Benjamins Publishing Company, 1992, p. 25-44.

— "Les verbes psychologiques". Langue française, 1995, 105, p. 6-15.

DELBECQUE, N. y B. LAMIROY. "Towards a typology fo the Spanish dative". En: VAN BELLE, W. y W. VAN LANGENDONCK (eds.). The Dative, volume 1, Descriptive Studies. Amsterdam, Philadelphia: John Benjamins, 1996.

DI TULLIO, Á. "Alternancia acusativo-dativo en verbos psicológicos del español". En: Atti del XXI Congresso di Linguistica e Filologia Romanza, Vol. II. Tübingen: Max Niemeyer Verlag, 1997.

DOWTY, D. "Thematic proto-roles and argument selection". Language, 1991, 67, p. 547-619.

FERNÁNDEZ ORDÓÑEZ, I. "Leísmo, laísmo y loísmo: estado de la cuestión". En: FERNÁNDEZ SORIANO, O. (ed.). Los pronombres átonos. Madrid: Taurus, 1993, p. 63-96.

— "Leísmo, laísmo y loísmo". En: BOSQUE, I. y V. DEMONTE, (eds.). Gramática descriptiva de la lengua española I. Madrid: Real Academia Española, Espasa Calpe, 1999, p. 1317-1393.

FERNÁNDEZ RAMÍREZ, S. Gramática española: 3.2. El pronombre. Madrid: Arco/Libros, 1987.

GARCÍA, É. The role of theory in linguistic analysis: The Spanish pronoun system. Amsterdam: North Holland Publishing Co, 1975.

- y R. OTHEGUY. "Dialect variation in leísmo: a semantic approach". En: FASOLD, R. y R. SHUY (eds.). Studies in language variation: Semantics, syntax, phonology, 
pragmatics, social situations, ethnographic approaches. Washington, DC: Georgetown University Press, 1977, p. 65-87.

HUYGENS, A. "El leísmo: una comparativa entre Sevilla y Madrid". Romanistik in Geschichte und Gegenwart, 2002, 8 (2), p. 199-213.

KLEIBER, G. La sémantique du prototype. Catégories et sens lexical. Paris: Presses Universitaires de France, 1990.

KLEIN-ANDREU, F. Variación actual y evolución histórica: los clíticos le/s, la/s, lo/s. München: Lincom, 2000.

LAPESA, R. "Sobre los orígenes y evolución del leísmo, laísmo y loísmo". En: BALDINGER, K. (ed.). Festschrift Walther von Wartburgzum 80. Geburtstag, vol. 2. Tübingen: Niemeyer, 1968, p. 523-551.

MARCOS MARÍN, F. Estudios sobre el pronombre. Madrid: Gredos, 1978.

PRIMUS, B. Cases and Thematic Roles. Tübingen: Niemeyer, 1999.

REAL ACADEMIA ESPAÑOLA. Esbozo de una nueva gramática de la lengua española. Madrid: Espasa-Calpe, 1973.

ROEGIEST, E. Variation actantielle de l' object direct et structure argumentale des verbes de perception en espagnol (inédito), 2000.

- Variación pronominal en español: el pronombre dativo entre sintaxis y semántica (inédito), 2001.

RUWET, N. Théorie syntaxique et syntaxe du français. Paris: Editions du Seuil, 1972.

- "Etre ou ne pas etre un verbe de sentiment". Langue française, 1994, 103, p. 45-55.

URUBURU BIDAURRÁZAGA, A. Estudios sobre leísmo, laísmo y loísmo. (Sobre el funcionamiento de los pronombres personales átonos o afijos no reflejos de $3^{a}$ persona, o de $2^{a}$ con cortesía). Córdoba: Universidad de Córdoba, 1993.

VAN VOORST, J. "A localist model for event semantics". Journal of Semantics, 1993, 10, p. 65-111.

— "Le contrôle de l'espace psychologique". Langue française, 1995, 105, p. 17-27.

VÁZQUEZ ROZAS, V. El complemento indirecto en español. Santiago de Compostela: Universidade de Santiago de Compostela, 1995. 